\title{
Stereotactic Radiotherapy Planning Using Modified Dynamic Conformal Arcs under Considering the Possibility for Amended Visual Organ Displacement Resulting from Early Tumor Shrinkage during Treatment for Perioptic Involvement of Myeloma
}

\author{
Kazuhiro Ohtakara1,2*, Hiroaki Hoshi² \\ ${ }^{1}$ Department of Radiation Oncology, Murakami Memorial Hospital, Asahi University, Gifu, Japan \\ ${ }^{2}$ Department of Radiology, Gifu University Graduate School of Medicine, Gifu, Japan \\ Email: ${ }^{*}$ ohtakara@murakami.asahi-u.ac.jp
}

Received 27 June 2014; revised 26 July 2014; accepted 20 August 2014

Copyright (C) 2014 by authors and Scientific Research Publishing Inc.

This work is licensed under the Creative Commons Attribution International License (CC BY).

http://creativecommons.org/licenses/by/4.0/

(c) (i) Open Access

\section{Abstract}

Treatment planning of radiotherapy for skull base involvement of multiple myeloma presenting with visual impairment should be optimized to alleviate symptoms immediately and sufficiently while minimizing toxicities. Two such patients were treated with fractionated stereotactic radiotherapy by using Dynamic Conformal Arcs (DCA) under image guidance based on bony anatomy alignment. DCA planning was optimized after considering the possibility for amendment of visual organ displacement resulting from early tumor shrinkage during treatment through 1) the use of a target volume with modified geometry as a surrogate for leaf adaptation in order to improve target coverage, and 2) manual adjustment of a subset of leaf positions to reduce the dose gradient immediately inside the target boundary facing the visual organs and to eliminate an undesirable dose hotspot. In both cases, anticipated geometric changes in the target volume associated with improvement of visual organ displacement toward the target centroid were observed before the completion of treatment. Favorable visual functional outcomes as well as local tumor control were achieved during 14 months and 4 months follow-up periods. Notably, inexorable visual loss in one patient was fully reversed within one month after radiotherapy. We described the modification

\footnotetext{
*Corresponding author.
}

How to cite this paper: Ohtakara, K. and Hoshi, H. (2014) Stereotactic Radiotherapy Planning Using Modified Dynamic Conformal Arcs under Considering the Possibility for Amended Visual Organ Displacement Resulting from Early Tumor Shrinkage during Treatment for Perioptic Involvement of Myeloma. International Journal of Medical Physics, Clinical Engineering and Radiation Oncology, 3, 183-192. http://dx.doi.org/10.4236/ijmpcero.2014.33024 
techniques for DCA planning in detail.

Keywords Multiple Myeloma, Interfractional Anatomical Change, Dynamic Conformal Arcs, Image-Guided
Radiotherapy, Stereotactic Radiotherapy

\section{Introduction}

Skull base involvement is an unusual clinical manifestation of symptomatic multiple myeloma (MM), and it can be the predominant site of either relapsed or initial disease [1] [2]. Owing to its proximity to a number of critical organs, patients with skull base involvement of MM can present with a wide spectrum of clinical presentations including impaired visual function [2].

Notwithstanding recent advances in pharmacotherapy and the intrinsically systemic nature of the disease, radiotherapy continues to have a key role in the treatment of patients with MM in order to control medically refractory tumors or to ameliorate symptoms including pain or epidural spinal cord compression [3] [4]. When radiotherapy is considered for symptomatic perioptic involvement of MM, treatment planning must be optimized to achieve expeditious and sufficient alleviation of symptoms as well as the preservation of visual function, whilst minimizing related toxicities and the treatment burden on the patients. In these regards, stereotactic irradiation is a promising approach [4]. Currently available image guidance systems can confer 6 degree-of-freedom (6D) verification and correction of daily patient setup errors [5], for which bony anatomy alignment is the mainstay for cranial stereotactic radiotherapy and is predicated on the premise that target volume geometry and its location relative to the cranium remain constant during the course of radiotherapy [5] [6]. However, in clinical practice, this premise may be compromised by early tumor shrinkage resulting in displacement of the compressed neighboring structures, especially in the case of radiosensitive tumors such as MM [6]. Amendment of the critical organ displacement toward the target centroid inevitably leads to a gradual dose increase to these organs in excess of the planned dose.

Herein, we describe two cases in which patients harboring skull base involvement of MM presented with visual impairment, and were treated by using image-guided fractionated stereotactic radiotherapy (SRT) based on modified dynamic conformal arcs (DCA) planning, with special consideration given to the possibility of visual organ displacement resulting from early tumor shrinkage during treatment. We describe the planning techniques and preliminary clinical results, including the magnitude of internal anatomical changes during treatment and relevant changes to the dosimetric parameters.

\section{Case Presentation}

\subsection{Case 1}

A 73-year-old woman experienced pathological fractures of the ribs and the left clavicle, and 4 months later, she was diagnosed with immunoglobulin G (IgG) kappa symptomatic MM (Durie-Salmon stage IIIA, International Staging System II). She had no remarkable past medical history. In addition to multiple pathological fractures, she had mild renal dysfunction and hypercalcemia. Initial imaging studies also revealed an asymptomatic sellar mass that was initially diagnosed as a cavernous sinus meningioma and managed conservatively. As an initial systemic treatment, 6 courses of bortezomib, melphalan, and prednisolone were administered. After the first course of treatment, she experienced slight diplopia and ptosis at the right side. Magnetic resonance (MR) images obtained 2 months after the initial study showed a slight enlargement of the sellar tumor, associated with increased compression of the optic chiasm (Figure 1(a) and Figure 2(d)). Although her visual symptoms were not progressive, early radiotherapy was considered in order to preserve visual function before the occurrence of tumor progression refractory to pharmacotherapy. SRT by using modified DCA was administered in 10 fractions with a total dose of 41.2 Gy to the isocenter (Table 1 and Table 2). Notably, MR images after the completion of 9 fractions (13 days after planning image acquisition) revealed clear shrinkage of the cranial portion of the tumor along with downward displacement of the optic chiasm (Figure 2(e)). As expected, the resulting change in 


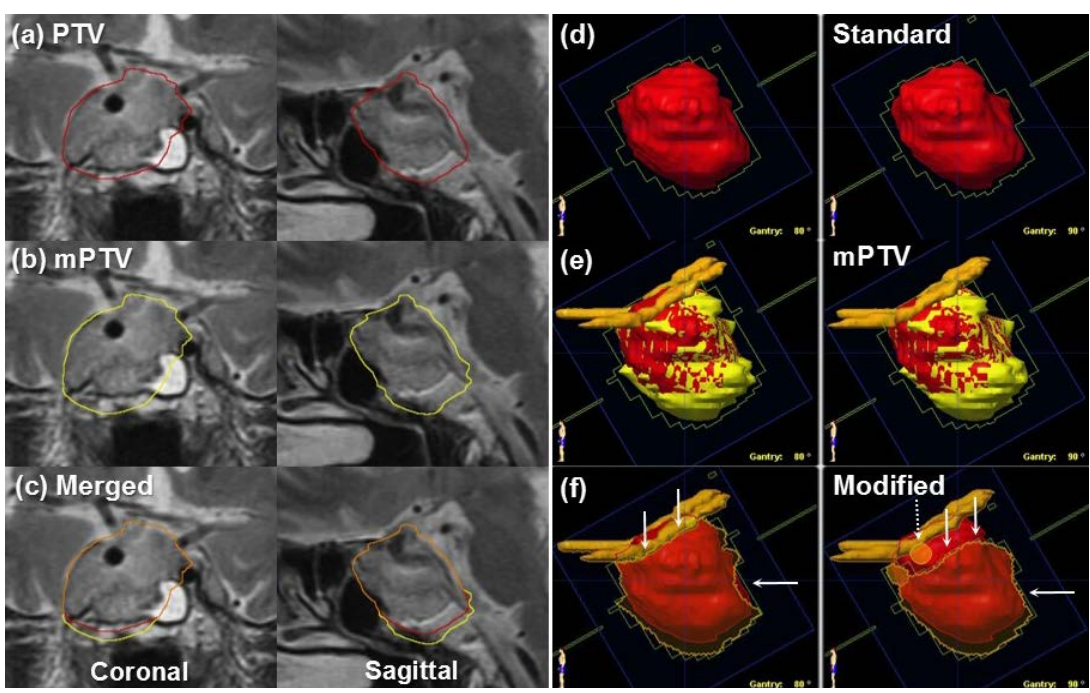

Figure 1. Target definition (a)-(c) and the method for leaf adaptation (d)-(f): Beam's eye views from a coplanar arc with the gantry angles of $80^{\circ}$ and $90^{\circ}$ in Case 1 . Original planning target volume (PTV, red) (a), modified PTV (mPTV, yellow) as a surrogate target for leaf adaptation (b), and merged image (orange, overlapped) (c). Note that the mPTV is generated by expanding the PTV caudally. Leaf adaptation to the PTV (red) with a $0.5 \mathrm{~mm}$ isotropic margin (d), and leaf fitting to the mPTV (yellow) with a $0.5 \mathrm{~mm}$ isotropic margin (e). The optic apparatus (OA) is also shown. Leaf adaptation to the mPTV with manual adjustment of a subset of leaf positions by using a cursor (dashed arrow) to reduce the dose to the OA and to reduce target over-coverage (arrows) in the actual plan (f).

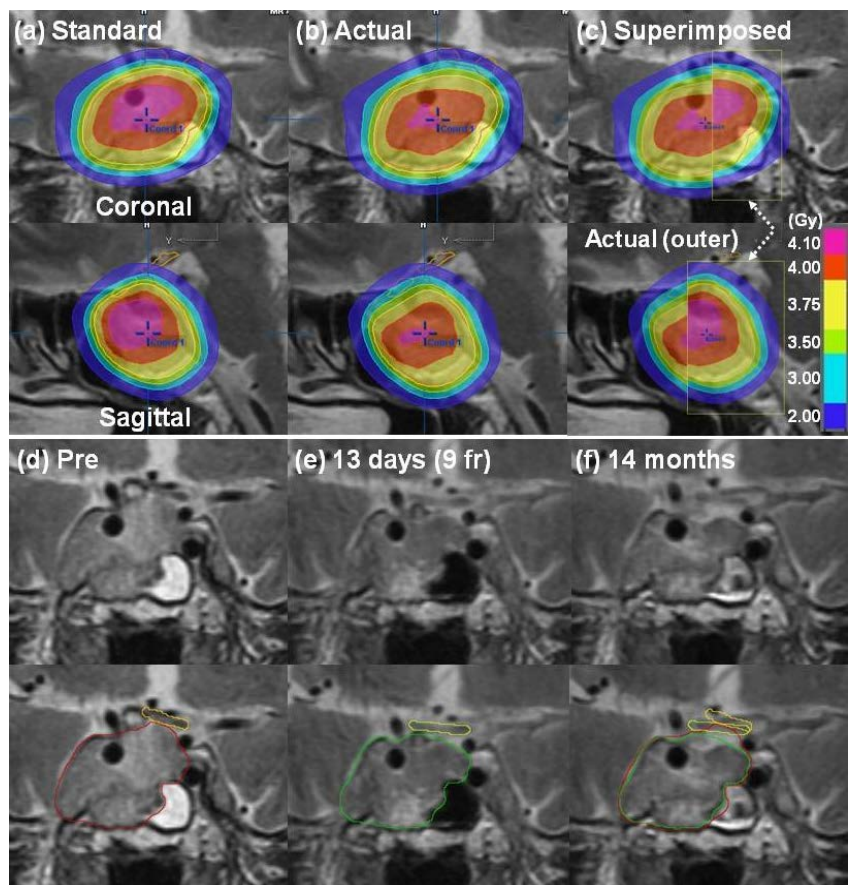

Figure 2. Dose distributions (a)-(c) and T2-weighted magnetic resonance images (T2-WI) before, during, and after radiotherapy (d)-(f) in Case 1. Absorbed doses are represented as dose per fraction (fr). Standard plan with the use of an isotropic leaf margin fitting to the modified planning target volume (mPTV) periphery (a). Note the higher dose area located in the cranial side within the PTV. Actually delivered plan with manual adjustment of leaf adaptation for the mPTV (b). Superimposed image (actually delivered plan [outer] versus standard plan [dashed arrows]) (c). Note the difference in and around the OA. Pre-treatment (d). Thirteen days after the start of radiotherapy (at 9 of 10 fractions delivered) (e). One year after radiotherapy (f). The PTV and the optic chiasm are contoured in each lower column (d), (e). The PTV and the optic chiasm before and 13 days after radiotherapy, respectively, are superimposed on the image taken 1 year later (f). Note the tumor shrinkage resulting in the downward displacement of the optic chiasm during treatment. 
Table 1. Basic arc arrangements for standard and modified (actually delivered) plans.

\begin{tabular}{ccccccc}
\hline & Arc number & Couch rotation $\left(^{\circ}\right)$ & Gantry rotation $\left(^{\circ}\right)$ & Collimator angle $\left(^{\circ}\right)$ & Weight & Leaf margin $(\mathrm{mm})^{\mathrm{a}}$ \\
\hline \multirow{3}{*}{ Case 1} & 1 & 0 & $210-330$ & 60 & 1.00 & 0.5 \\
& 2 & 295 & $210-330$ & 10 & 1.00 & 0.5 \\
& 3 & 60 & $30-150$ & 10 & 1.00 & 0.5 \\
& 4 & 0 & $30-150$ & 300 & 1.00 & 0.5 \\
\hline \multirow{2}{*}{ Case 2} & 1 & 0 & $210-330$ & 0 & 1.00 & 1.0 \\
& 2 & 305 & $210-330$ & 0 & 1.00 & 1.0 \\
& 3 & 85 & $30-150$ & 0 & 1.00 & 1.0 \\
\hline
\end{tabular}

${ }^{\mathrm{a}} \mathrm{A}$ subset of leaf positions is manually adjusted for modified plans.

Table 2. Dosimetric parameters: Comparison between standard versus modified plans and the changes during treatment (Pre versus Post).

\begin{tabular}{|c|c|c|c|c|c|c|c|c|}
\hline & \multirow{2}{*}{ Object } & \multirow{2}{*}{ Parameters } & \multicolumn{3}{|c|}{ Standard } & \multicolumn{3}{|c|}{ Modified (Actual) } \\
\hline & & & Pre & Post & Change & Pre & Post & Change \\
\hline \multirow{8}{*}{ Case 1} & \multirow{5}{*}{ PTV } & Volume (cc) & 15.1 & 13.9 & $-7.9 \%$ & 15.1 & 13.9 & $-7.9 \%$ \\
\hline & & D98/fr (Gy) & 3.39 & 3.43 & $+1.2 \%$ & 3.30 & 3.41 & $+3.3 \%$ \\
\hline & & D50/fr (Gy) & 4.03 & 4.04 & $+0.2 \%$ & 4.00 & 4.00 & NC \\
\hline & & D2/fr (Gy) & 4.18 & 4.18 & NC & 4.13 & 4.13 & NC \\
\hline & & HI & 0.20 & 0.19 & -0.01 & 0.21 & 0.18 & -0.03 \\
\hline & \multirow{3}{*}{ Optic apparatus } & Dmax/fr (Gy) & 4.00 & 4.12 & $+3.0 \%$ & 3.66 & 3.69 & $+0.8 \%$ \\
\hline & & $\mathrm{D}_{0.05 \mathrm{cc}} / \mathrm{fr}(\mathrm{Gy})$ & 3.82 & 3.96 & $+3.7 \%$ & 3.37 & 3.59 & $+6.5 \%$ \\
\hline & & $\mathrm{D}_{0.1 \mathrm{cc}} / \mathrm{fr}$ (Gy) & 3.68 & 3.88 & $+5.4 \%$ & 3.24 & 3.50 & $+8.0 \%$ \\
\hline \multirow{21}{*}{ Case 2} & \multirow{5}{*}{$\begin{array}{c}\text { PTV1 } \\
\text { (Sphenoid) }\end{array}$} & Volume (cc) & 45.8 & 28.8 & $-37.1 \%$ & 45.8 & 28.8 & $-37.1 \%$ \\
\hline & & D98/fr (Gy) & 3.68 & 3.75 & $+1.9 \%$ & 3.69 & 3.62 & $-1.9 \%$ \\
\hline & & D50/fr (Gy) & 4.11 & 4.12 & $+0.2 \%$ & 4.05 & 4.00 & $-1.2 \%$ \\
\hline & & D2/fr (Gy) & 4.52 & 4.50 & $-0.4 \%$ & 4.40 & 4.41 & $+0.2 \%$ \\
\hline & & HI & 0.20 & 0.18 & -0.02 & 0.18 & 0.20 & +0.02 \\
\hline & \multirow{5}{*}{$\begin{array}{c}\text { PTV2 } \\
\text { (Subtemporal) }\end{array}$} & Volume (cc) & 7.3 & 3.7 & $-49.3 \%$ & 7.3 & 3.7 & $-49.3 \%$ \\
\hline & & D98/fr (Gy) & 4.16 & 4.38 & $+5.3 \%$ & 3.88 & 4.33 & $+11.6 \%$ \\
\hline & & D50/fr (Gy) & 4.59 & 4.64 & $+1.1 \%$ & 4.43 & 4.46 & $+0.7 \%$ \\
\hline & & D2/fr (Gy) & 4.77 & 4.78 & $+0.2 \%$ & 4.53 & 4.55 & $+0.4 \%$ \\
\hline & & $\mathrm{HI}$ & 0.13 & 0.09 & -0.04 & 0.15 & 0.05 & -0.10 \\
\hline & \multirow{5}{*}{$\begin{array}{l}\text { PTV3 }^{\mathrm{a}} \\
\text { (Clivus) }\end{array}$} & Volume (cc) & 19.7 & $\mathrm{NE}$ & $\mathrm{NE}$ & 19.7 & $\mathrm{NE}$ & $\mathrm{NE}$ \\
\hline & & D98/fr (Gy) & 2.96 & $\mathrm{NE}$ & $\mathrm{NE}$ & 3.14 & $\mathrm{NE}$ & $\mathrm{NE}$ \\
\hline & & D50/fr (Gy) & 3.41 & $\mathrm{NE}$ & $\mathrm{NE}$ & 3.39 & $\mathrm{NE}$ & $\mathrm{NE}$ \\
\hline & & D2/fr (Gy) & 3.67 & $\mathrm{NE}$ & $\mathrm{NE}$ & 3.60 & $\mathrm{NE}$ & $\mathrm{NE}$ \\
\hline & & $\mathrm{HI}$ & 0.21 & $\mathrm{NE}$ & $\mathrm{NE}$ & 0.14 & $\mathrm{NE}$ & $\mathrm{NE}$ \\
\hline & \multirow{3}{*}{ Optic nerve } & Dmax/fr (Gy) & 3.88 & 4.10 & $+5.7 \%$ & 3.7 & 4.00 & $+8.1 \%$ \\
\hline & & $\mathrm{D}_{0.05 \mathrm{cc}} / \mathrm{fr}(\mathrm{Gy})$ & 3.81 & 4.04 & $+6.0 \%$ & 3.47 & 3.93 & $+13.3 \%$ \\
\hline & & $\mathrm{D}_{0.1 \mathrm{cc}} / \mathrm{fr}$ (Gy) & 3.77 & 4.01 & $+6.4 \%$ & 3.42 & 3.87 & $+13.2 \%$ \\
\hline & \multirow{3}{*}{ Retina } & Dmax/fr (Gy) & 4.12 & 4.26 & $+3.4 \%$ & 3.87 & 4.08 & $+5.4 \%$ \\
\hline & & $\mathrm{D}_{0.05 c \mathrm{c}} / \mathrm{fr}(\mathrm{Gy})$ & 3.86 & 4.16 & $+7.8 \%$ & 3.60 & 3.96 & $+10.0 \%$ \\
\hline & & $\mathrm{D}_{0.1 \mathrm{cc}} / \mathrm{fr}$ (Gy) & 3.69 & 4.07 & $+10.3 \%$ & 3.47 & 3.87 & $+11.5 \%$ \\
\hline
\end{tabular}

PTV: planning target volume; Dn: (\%) represents the dose (Gy) receiving at least n\% of a reference volume; fr: fraction; NC: no change; HI: homogeneity index; Dmax: maximum dose; NE: not evaluated; Dncc: (\%) means the dose (\%) receiving at least $\mathrm{n} \mathrm{cm}^{3}$ of a reference volume. Homogeneity index is defined as (D2-D98)/D50 according to Report 83 of the International Commission on Radiation Units and Measurements (ICRU) [8]. ${ }^{\mathrm{a}}$ The PTV3 was the clivus involvement without extraosseous extension, and no significant volume change during treatment was observed. 
the optic chiasm dose was deemed acceptable (Table 2), and the planned SRT was completed as scheduled. Sustained tumor control was achieved 14 months after SRT (Figure 2(f)), and her visual symptoms resolved without any apparent SRT-related acute or late adverse reactions at the last follow-up visit. Systemic control of MM was still maintained 18 months after initial treatment through the continued administration of lanalidomide and dexamethasone.

\subsection{Case 2}

A 54-year-old man, with his past medical history being unremarkable, complained of multiple bone pain and subsequent dyspnea that was attributable to pneumonitis and pleural effusion. He also had renal dysfunction, anemia, hyperuricemia, and hypercalcemia, and he was diagnosed with IgG kappa symptomatic MM (DurieSalmon stage IIIA, International Staging System II) 3 months after experiencing the initial symptoms. Steroids were administered to treat the MM-related pneumonitis, resulting in clinical improvement. Subsequently, 6 courses of lanalidomide and dexamethasone were administered, resulting in an initial response, but the patient showed a relapse 6 months after the commencement of treatment. One month before the clinical relapse, he noticed impaired visual acuity in the right eye as well as diplopia. Subsequently, his visual symptoms worsened to inexorable visual loss (only slight light perception), chemosis, and proptosis with difficulty in eyelid closure due to swelling in the right orbito-temporal region and mild perioral numbness. Imaging studies revealed that these symptoms were attributable to skull base involvement of MM, particularly to a mass located in and around the sphenoidal ridge, extending into the subtemporal space, and involving the clivus (Figure 3 and Figure 4(a)).

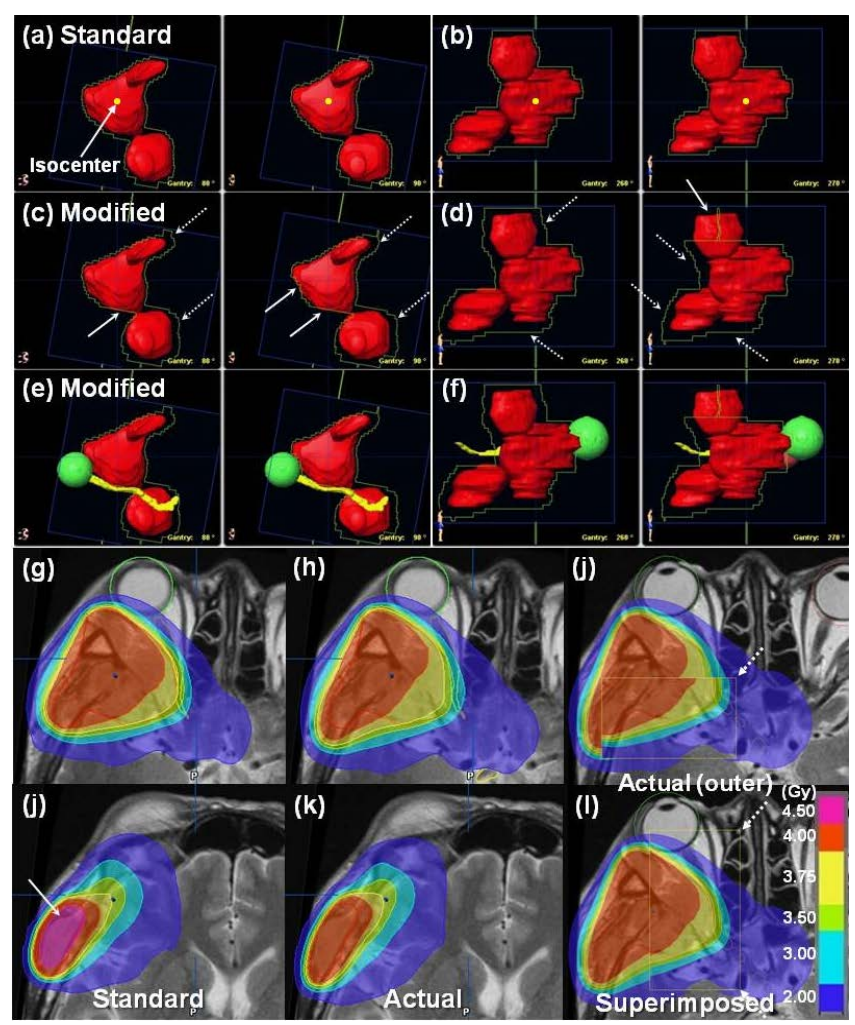

Figure 3. The method for leaf adaptation (a)-(f): Beam's eye views and representative dose distributions (g)-(l) in Case 2. A non-coplanar arc with the couch angle of $85^{\circ}$ and the gantry angles of $80^{\circ}$ and $90^{\circ}$ (a), (c), (e). A coplanar arc with the gantry angles of $260^{\circ}$ and $270^{\circ}$ (b), (d), (f). Standard planning: Leaf adaptation to the PTV (red) by using an isotropic margin (a), (b). Actual planning: leaf adaptation to the mPTV (not shown) with manual adjustment of a subset of leaf positions to reduce the dose to the optic apparatus (OA) and the subtemporal lesion (arrows) as well as to improve target coverage (dashed arrows) (c)-(f). The right eyeball and the OA are superimposed (e), (f). Standard plan with the use of an isotropic margin fitting to the mPTV actually delivered plan (h), (k). Superimposed image (dose distributions in the standard plan are displayed in the spyglass view [dashed arrows]) (j), (l). Note that the highest dose area in the subtemporal lesion (arrow) as well as the dose to the retina and the optic nerve in the standard plan is mitigated in the actually delivered plan. 
Systemic treatment comprising bortezomib, cyclophosphamide, and dexamethasone was immediately administered the day after diagnosis. In addition, SRT was administered the day after he was referred to our department. Although numerous punched-out lesions in the calvarium were evident, SRT for the predominant skull base lesions (sphenoidal ridge, subtemporal, and clivus) was administered with a planned total dose of $40 \mathrm{~Gy}$ in 10 fractions to the isocenter set to the centroid of the sphenoidal ridge lesion, with simultaneous irradiation of these 3 adjacent targets via a single isocenter [7]. Notably, computed tomography (CT) images obtained 11 days after the commencement of FSRT (completion of 8 of 10 fractions) showed significant tumor shrinkage concomitant with displacement of the visual organ towards the isocenter (Figure 4(e), Figure 4(f)). SRT was discontinued after the completion of only 9 of 10 fractions owing to the unexpectedly high dose to the normal tissues including the optic nerve and the retina (Table 2). One week after the commencement of SRT, the patient noticed a significant improvement in visual acuity and reduced diplopia, and his symptoms related to skull base involvement had almost disappeared within one month of treatment. Acute radiation-related toxicity included grade 1 dermatitis and conjunctivitis limited extraordinarily to the right outer canthus. Cerebrospinal fluid (CSF) examination 18 days after the initiation of SRT revealed MM involvement, and intrathecal administration of prednisolone, methotrexate, and cytarabine was therefore commenced. Four months after SRT, he retained normal visual acuity and field without chemosis, proptosis, or periorbital swelling, and sustained tumor regression was apparent on imaging surveillance. Systemic control of MM including central nervous system (CNS) involvement was also maintained through continued systemic treatment.

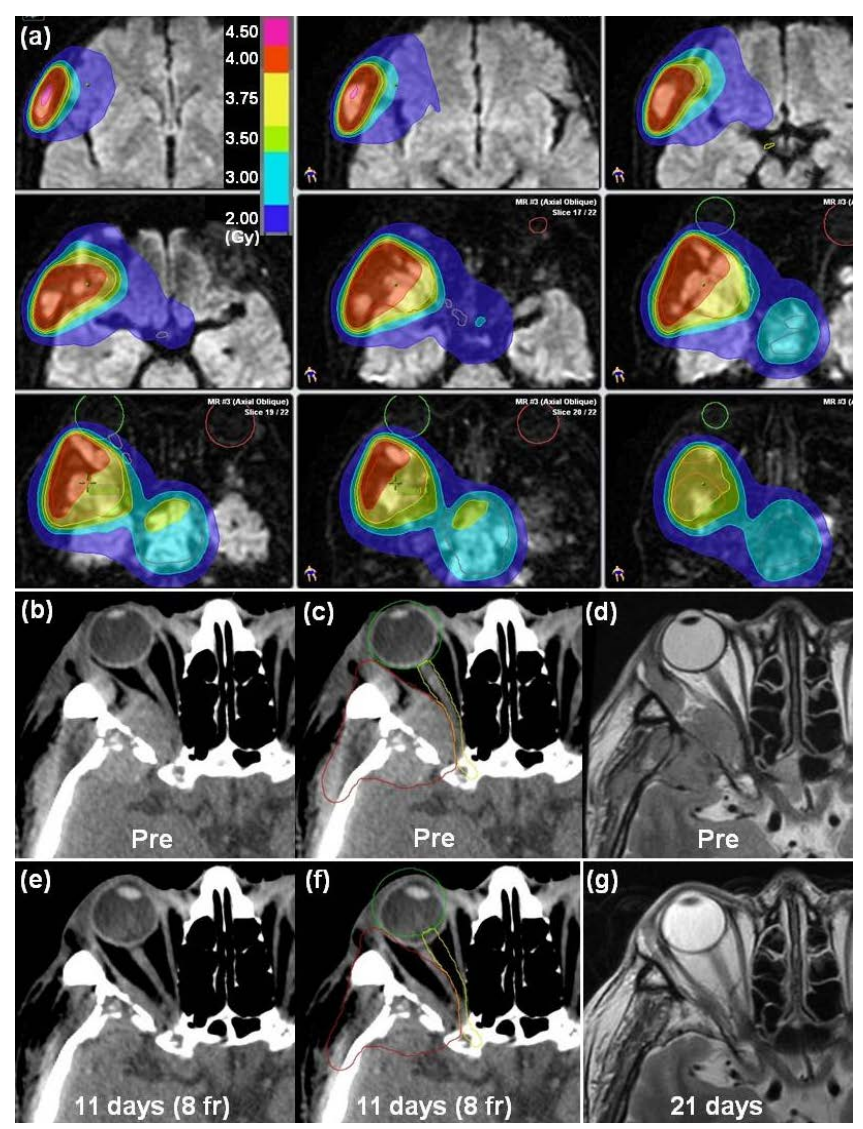

Figure 4. Dose distributions on diffusion-weighted images almost in its entirety, (a) and planning computed tomography (CT) images and T2-weighted images (T2-WI) before radiotherapy (b)-(d, Pre) and 11 days (e), (f) and 21 days (g) after the commencement of radiotherapy, respectively, in Case 2. Note the optic nerve (ON) displacement medially caused by the tumor, while the boundary between the tumor and the ON can be deciphered through the distinct intensities (d). The planning target volume (PTV), the eyeball, and the ON contoured on the pre-treatment images (c) are superimposed onto the 11-day CT image (f). Note the marked shrinkage of the tumor associated with significant displacement of the eyeball and the ON toward the tumor centroid. T2-WI taken 21 days after radiotherapy showing further tumor shrinkage, leading to sufficient decompression of the ON (g). 


\section{Treatment Planning and Delivery Methods}

SRT was delivered through the DCA technique by using the NovalisTx ${ }^{\mathrm{TM}}$ platform (BrainLAB AG, Feldkirchen, Germany and Varian Medical Systems, Palo Alto, CA, USA) via patient immobilization with a general thermoplastic mask [5]. iPlan Image 4.1 ${ }^{\mathrm{TM}}$ (BrainLAB AG) was used for image co-registration and object contouring, and iPlan RT Dose 4.1.2. ${ }^{\mathrm{TM}}$ (BrainLAB AG) was used for treatment planning. Planning CT scans were acquired in contiguous $1.25 \mathrm{~mm}$ slices and fused to post-contrast T1-weighted MR images along with T2- and diffusion-weighted images. In both cases, the gross tumor volumes (GTVs) directly compressed the visual organs i.e. the optic chiasm in Case 1, and the optic nerve and the eyeball in Case 2. Furthermore, there were 3 symptomatic contiguous or adjacent GTVs in Case 2 (Figure 4(a)). The clinical target volumes (CTVs) were delineated by referring to all MR images in order to ensure that the CTV fully encompassed the GTV (Figure 1(a) and Figure 4(c)). The planning target volumes (PTVs) were set identical to the CTVs, given the proximity of the GTVs to the optic apparatus. The DCA plans consisted of 4 arcs including one or two coplanar arcs to shorten the treatment time. The details for arc/collimator settings are described in Table 1. Standard planning was based on leaf fitting to the "PTV" per se with the use of an "isotropic" leaf margin to the PTV periphery to ensure that the PTV was encompassed by $85 \%$ and $87.5 \%$ isodose surfaces, normalized to $100 \%$ at the isocenter, for Cases 1 and 2, respectively. Assigned isocenter doses for Cases 1 and 2 were 41.2 Gy and 40.0 Gy, respectively, both in 10 fractions. The 85\% and 87.5\% isodoses in Cases 1 and 2, respectively, were therefore identical as both $35.0 \mathrm{~Gy}$. Thus, a relatively homogeneous target dose distribution for stereotactic irradiation was the objective in both cases [8]. The dose calculation algorithm was a pencil beam in conjunction with the radiological path length for tissue heterogeneity correction, with a grid size of $1 \mathrm{~mm}$. These standard plans were further modified by using the methods described below.

\subsection{The Use of a Modified Target in Lieu of the Original Target as a Surrogate for Leaf Adaptation}

Dose distribution resulting from DCA probably does not sufficiently cover the caudal side of the target (the ventral and dorsal sides in some cases), especially for homogeneous target dose planning [8]. Furthermore, treatment plans based on an isotropic leaf margin frequently have sub-optimal dose conformity [5]. To improve target coverage, a copy of PTV, referred to here as "modified PTV (mPTV)", was generated by adding a "0 mm" margin to the PTV, and it was used as a surrogate target for leaf adaptation, instead of the original PTV. On the basis of the fitting of a reference isodose surface to the PTV boundary, a part of the mPTV contour was manually modified (usually expanded), and the dose was re-calculated (Figure 1(b)). This process was reiterated as necessary until sufficient target coverage with acceptable dose conformity was attained (Figure 1 and Figure 3).

\subsection{Manual Adjustment of a Subset of Leaf Positions}

As a next step, a subset of leaf positions were manually adjusted on beam's eye views as illustrated in Figure 1(f), Figure 3(c), and Figure 3(d) to further optimize dose distribution through sufficient dose sparing to the visual organs, further amendment of target coverage (both under- and over-coverage), and a reduction in the steep dose increase just interior to the PTV boundary facing the visual organs (mitigation of the dose hotspot usually located at the cranial side within the target) [9]. This technique was also applied to attenuate the dose differences among multiple targets through attenuation of a dose hotspot at the superficially and cranially located target (PTV2), in Case 2 in which simultaneous irradiation of 3 contiguous/adjacent targets was performed via a single isocenter (Figure $3(\mathbf{j})$ and Figure $3(\mathbf{k})$ ). This process was also repeated as often as required. The degree of adjustment was limited to ensure a sufficient distance between the leaf-end positions for adjusted leaves and the isocenter, and to obviate the extremely irregular shape of the beam apertures.

Thus, the uses of a modified target as a surrogate for leaf adaptation and manually optimized anisotropic (nonisotropic) leaf margins to the target periphery were the basis of the modification techniques.

\section{Evaluation of Changes in Internal Anatomy and Dosimetric Parameters during Treatment}

Daily image guidance was implemented via 6D verification and correction on the basis of paired orthogonal $\mathrm{kV}$ radiographic images obtained by using the ExacTrac Robotics ${ }^{\circledR}$ (BrainLAB AG) equipped with the NovalisTx 
platform [5]. Before the completion of planned treatment, non-contrast CT scans or MR images were acquired at the time of administration of the 9th and 8th fractions in Cases 1 and 2, respectively, and they were fused to the planning CT images to evaluate any change of the tumor geometry and the visual organ displacement, and the resulting dosimetric parameters on the basis of a comparison of the standard and actually delivered plans. The PTV and the visual organs were re-contoured, and changes in the dosimetric parameters during SRT in the standard and actually delivered modified plans were evaluated (Table 2). Significant dosimetric changes of both the tumors and the visual organs were found for both the standard and actually delivered plans, whereas the doses to the visual organs through the course of SRT in standard plans were generally higher than those in the actual plans (Table 2).

\section{Discussion}

Dose distribution of stereotactic irradiation is intrinsically characterized by an inhomogeneous target dose, thereby achieving a steeper dose falloff outside the target boundary to minimize normal tissue toxicities as well as a sharper dose increases inside the target periphery to augment efficacy [8]. However, some of these principles may be compromised by administering SRT for $>1$ week for some radiosensitive tumors, namely, early tumor response during treatment [6]. This in turn can result in a gradual increase in the target dose, whilst concomitant displacement of the compressed visual structures toward the target centroid inevitably leads to an undesirable dose increase in the visual organs. A radiation dose in excess of the already planned dose may lead to a more expeditious and sufficient decompression of the visual organs through an early tumor response, whereas higher doses to the optic apparatus may compromise recovery from optic nerve dysfunction and also increase the risk of delayed optic neuropathy. Thus, the dose actually absorbed by the target and surrounding tissues in inhomogeneous target dose planning is probably susceptible to potential geometrical change and/or deviation of both the tumors and surrounding tissues during treatment [6]. Accordingly, homogeneous dose planning (85\% and 87.5\% isodose surfaces for coverage) was chosen in the cases described here. In order to generate conformal plans that fully spare the abutting visual organs, DCA planning was optimized by using the above-mentioned modification techniques. In addition, in order to reduce the excess dose to the visual organs during the course of SRT, we also considered the possibility of visual organ displacement during treatment and reduced the gradient of the dose increase immediately interior to the target boundary facing the visual organ via manual adjustment of a subset of the relevant leaf positions. We intended to develop DCA plans that were acceptable and tolerable in terms of cumulative dosage to the visual organs, even when the visual organ gradually displaced toward the target centroid during SRT. Early tumor response associated with amendment of the visual organ compression was documented in both cases, although significant displacement of the visual organs beyond anticipation was observed in Case 2.

Limited previous reports have described the concept and the methods for solving the SRT planning adaptation required after considering the possible displacement of the adjacent normal structures resulting from early tumor responses during treatment. Nonetheless, imaging surveillance until the completion of FSRT should be considered for these radiosensitive malignancies in order to evaluate any internal anatomical change and the resulting dosimetric changes, and to appropriately weigh the clinical outcomes against the planned dose distributions.

DCA is the mainstay for stereotactic irradiation of brain and head and neck lesions in our institutions. Because DCA can be commenced more quickly than intensity-modulated radiotherapy, it is suitable for oncological emergency cases, such as those described here. Furthermore, compared to static multi-beams, the relatively short treatment time also reduces the burden on patients, thereby minimizing intrafractional motion, which is preferable in frameless image-guided treatment [5]. DCA plans generated by setting an isotropic leaf margin still allow for amendments in dose conformity as well as to provide a sufficient dose reduction for the abutting critical organs. In order to overcome these shortcomings, the modified DCA planning described above has been applied to the vast majority of cases at our institute, excluding small spherical lesions [5].

A number of intrinsic study limitations must be considered. First, histopathological diagnoses of the skull base lesions were not obtained in both cases, although the clinical courses were deemed consistent with that of skull base involvement of MM on the basis of imaging findings and radiosensitivity. Second, the follow-up periods were short, and therefore, the validity of the dose fractionation schemes and the planning techniques used for both cases must be assessed by further clinical observation. In addition, regarding radiotherapy target definitions, there is a possibility that skull base involvement of MM may also indicate CNS involvement including 
CSF, as observed in Case 2, which is generally associated with a dismal prognosis [3] [10]. Accordingly, some practitioners may still prefer wide field conventional irradiation to fully encompass the subclinical disease beyond the overt lesion. In fact, conventional radiotherapy by using wide margin to the GTV was applied to most reported MM patients with perioptic skull base involvement [1]-[3], whereas there were very few reports pertaining to stereotactic irradiation as a sole radiotherapy method for these patients [4] [11] [12]. However, conventional dose fractionation schemes by using doses such as 1.8 - 2 Gy per fraction may be disadvantageous in terms of how quickly and efficiently they can alleviate relevant symptoms. Furthermore, wide field irradiation may lead to further burden on patients in terms of relevant additional acute toxicities which might be augmented by chemotherapeutic agents. For oncological emergency cases, i.e. patients with relentlessly progressive visual symptoms, such as those exhibited in Case 2, expeditious and sufficient decompression of the affected organs would be preferable. On the other hand, in an extremely hypofractionated regimen with the use of $\leq 5$ fractions, sufficient dose administration is substantially compromised by the dose constraint for the visual organs [4] [12]. Therefore, extremely hypofractionation or even single fraction regimens are deemed unsuitable in these clinical scenarios. In this regard, the intermediary-dose hypofractionated regimen used for both of the cases described here appeared to achieve a suitable balance between efficacy and safety. Nonetheless, further investigations are needed to validate whether the presented fractionated SRT using modified DCA techniques can confer better clinical outcomes in terms of durable local control and minimal toxicities compared to conventional radiotherapy or extremely hypofractionated SRT.

In conclusion, through the illustration of these cases, we described the concept and the techniques needed for treatment planning after considering the possibility of early tumor response and the resulting displacement of compressed critical structures during SRT by using DCA for radiosensitive malignancies like MM.

\section{Conflict of Interest}

None.

\section{References}

[1] Joshi, A., Jiang, D., Singh, P. and Moffat, D. (2011) Skull Base Presentation of Multiple Myeloma. Ear, Nose, \& Throat Journal, 90, E6-E9.

http://connection.ebscohost.com/c/case-studies/70247861/skull-base-presentation-multiple-myeloma

[2] Wachter, D., Böker, D.K., Huegens-Penzel, M., Kuchelmeister, K. and Jödicke, A. (2010) First Manifestation of Lambda Positive Plasmacytoma in the Orbital Apex with Acute Unilateral Loss of Vision. Minimally Invasive Neurosurgery, 53, 74-76. https://www.thieme-connect.com/DOI/DOI?10.1055/s-0030-1249051 http://dx.doi.org/10.1055/s-0030-1249051

[3] Chen, C.I., Masih-Khan, E., Jiang, H., et al. (2013) Central Nervous System Involvement with Multiple Myeloma: Long Term Survival Can Be Achieved with Radiation, Intrathecal Chemotherapy, and Immunomodulatory Agents. British Journal of Haematology, 162, 483-488. http://onlinelibrary.wiley.com/doi/10.1111/bjh.12414/full http://dx.doi.org/10.1111/bjh.12414

[4] Wong, E.T., Lu, X.Q., Devulapalli, J. and Mahadevan, A. (2006) Cyberknife Radiosurgery for Basal Skull Plasmacytoma. Journal of Neuroimaging, 16, 361-363. http://onlinelibrary.wiley.com/doi/10.1111/j.1552-6569.2006.00062.x/full

[5] Ohtakara, K., Hayashi, S., Tanaka, H., et al. (2012) Clinical Comparison of Positional Accuracy and Stability between Dedicated versus Conventional Masks for Immobilization in Cranial Stereotactic Radiotherapy Using 6-Degree-ofFreedom Image Guidance System-Integrated Platform. Radiotherapy and Oncology, 102, 198-205. http://www.sciencedirect.com/science/article/pii/S0167814011006244 http://dx.doi.org/10.1016/j.radonc.2011.10.012

[6] Ohtakara, K. and Hoshi, H. (2014) Target Volume Geometric Change and/or Deviation from the Cranium during Fractionated Stereotactic Radiotherapy for Brain Metastases: Potential Pitfalls in Image Guidance based on Bony Anatomy Alignment. Journal of Medical Imaging and Radiation Oncology, in Press. http://dx.doi.org/10.1111/1754-9485.12194

[7] Roberge, D., Ruo, R. and Souhami, L. (2006) Killing Two Birds with One Stone: A Dosimetric Study of Dual Target Radiosurgery Using a Single Isocenter. Technology in Cancer Research and Treatment, 5, 613-617. http://www.tcrt.org/c3025/c4218/Killing-Two-Birds-with-One-Stone-A-Dosimetric-Study-of-Dual-Target-Radiosurger y-Using-a-Single-Isocenter-p-613-618-p15413.html

[8] Ohtakara, K., Hayashi, S., Tanaka, H. and Hoshi, H. (2012) Consideration of Optimal Isodose Surface Selection for 
Target Coverage in Micro-Multileaf Collimator-Based Stereotactic Radiotherapy for Large Cystic Brain Metastases: Comparison of 90\%, 80\% and 70\% Isodose Surface-Based Planning. The British Journal of Radiology, 85, e640-e646. http://www.birpublications.org/doi/full/10.1259/bjr/21015703 http://dx.doi.org/10.1259/bjr/21015703

[9] Ohtakara, K. and Hoshi, H. (2014) Cerebral Cyst Formation Following Stereotactic Ablative Irradiation for Non-Nasopharyngeal Head and Neck Malignancies: Imaging Findings and Relevant Dosimetric Parameters. The British Journal of Radiology, 87, Article ID: 20140071. http://www.birpublications.org/doi/full/10.1259/bjr.20140071 http://dx.doi.org/10.1259/bjr.20140071

[10] Yeung, S.N., Paton, K.E., Dorovini-Zis, K., Chew, J.B. and White, V.A. (2008) Histopathologic Features of Multiple Myeloma Involving the Optic Nerves. Journal of Neuro-Ophthalmology, 28, 12-16. http://dx.doi.org/10.1097/WNO.0b013e3181675457

[11] Peker, S., Abacioğlu, U., Bayrakli, F., Kiliç, T. and Pamir, M.N. (2005) Gamma Knife Radiosurgery for Cavernous Sinus Plasmacytoma in a Patient with Breast Cancer History. Surgical Neurology, 63, 174-176.

http://www.sciencedirect.com/science/article/pii/S0090301904000862 http://dx.doi.org/10.1016/j.surneu.2003.12.013

[12] Alafaci, C., Grasso, G., Conti, A., Caffo, M., Salpietro, F.M. and Tomasello, F. (2014) Cyberknife Radiosurgery for Cranial Plasma Cell Tumor. Turkish Neurosurgery, 24, 272-275.

http://www.turkishneurosurgery.org.tr/pdf/pdf_JTN_1311 
Scientific Research Publishing (SCIRP) is one of the largest Open Access journal publishers. It is currently publishing more than 200 open access, online, peer-reviewed journals covering a wide range of academic disciplines. SCIRP serves the worldwide academic communities and contributes to the progress and application of science with its publication.

Other selected journals from SCIRP are listed as below. Submit your manuscript to us via either submit@scirp.org or Online Submission Portal.
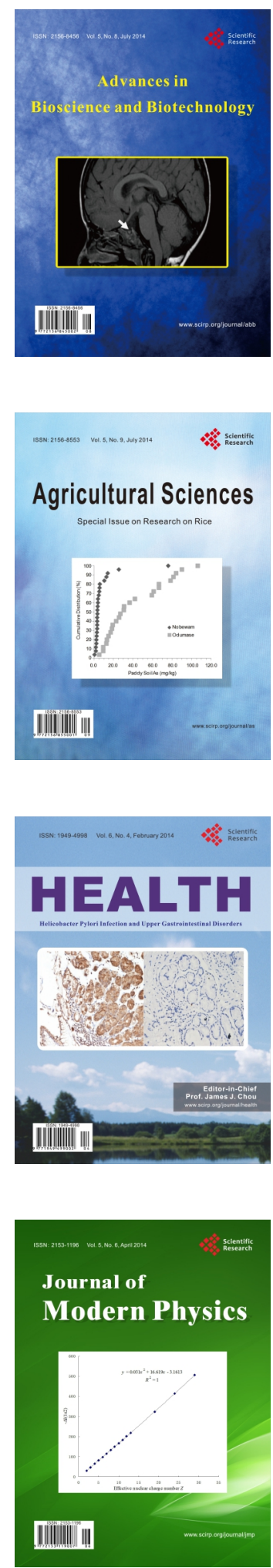
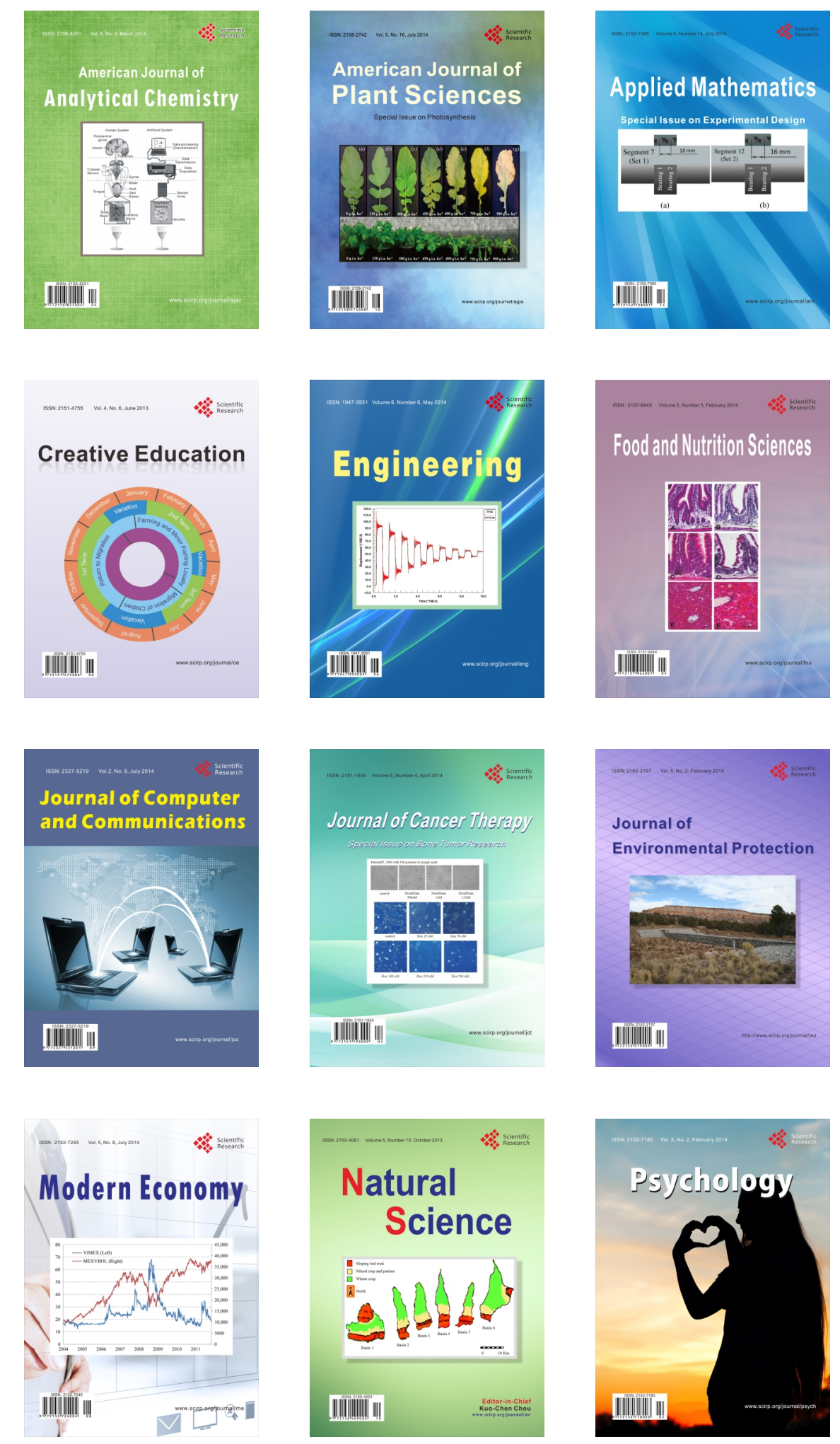